Cite this: RSC Adv., 2014, 4, 1168

\title{
High pressure synthesis of FePt nanoparticles with controlled morphology and Fe content $\uparrow$
}

\author{
Luke Alexander Wormell Green ${ }^{\mathrm{ab}}$ and Nguyễn Thi Kim Thanh*ab
}

Magnetic nanoparticles (MNPs) are intensively researched due to their high potential in biomedicine, catalysis, and high density information storage. FePt NPs could be an alternative for commonly used magnetite NPs and the synthesis of FePt NPs is an active area of research. The challenge is to increase the Fe content and saturation magnetisation of FePt NPs so that they can be used in many practical applications. Fine tuning of synthetic methods is required in order to achieve the enhanced magnetic properties of FePt nanoparticles and novel methods are being sought. Herein, use of an autoclave is shown to increase the Fe content, crystallinity and the subsequent magnetic properties of FePt pseudo cube nanoparticles compared to those synthesised under atmospheric pressure. Decreasing the amount of oleic acid is also shown to increase the iron content and can lead to elongated FePt nanoparticles under normal pressure. Further application of nanoparticles synthesised in organic media often requires functionalisation or exchange of stabiliser chemicals. Greater demand for control over such functionalisation requires more information about nanoparticle-stabiliser chemical interactions. Infra-red studies indicate mono and bidentate coordination with oleic acid, however shifts of spectra show that the strength of the bidentate interactions weaken with increasing oleic acid amount.

Received 8th October 2013

Accepted 1st November 2013

DOI: 10.1039/c3ra45664a

www.rsc.org/advances
Thermal decomposition of iron pentacarbonyl and polyol reduction of iron acetylacetonate methods are commonly used for synthesis of FePt nanoparticles. FePt NPs with spherical, ellipsoidal, cubic, rod shaped, wire and hexagonal morphology ranging from $2-20 \mathrm{~nm}$ in diameter are all possible. ${ }^{13-16}$ Nanoparticle shape is important as it can impact on the catalytic activity or biological effectiveness of NPs. For example catalysis is sensitive to shape, and reaction rates, activity and selectivity can all be tuned by varying the shape of the catalyst NP. ${ }^{17}$ Biologically, changes in shape will affect the amount of drag exerted by a NP and the lift required by blood flow for effective transport, meaning different shapes will likely interact with biological media in different ways. ${ }^{18}$

Traditionally MNPs have been synthesised under atmospheric pressure. In a drive to develop novel materials, new heating vessels such as autoclaves have been employed to make MNPs with novel morphology. ${ }^{19}$ Synthesis in autoclaves offers the opportunity to examine the effect of high pressure on the properties of NPs. Chemical precipitation of magnetite followed by heating in an autoclave has been shown to reduce the mean size and improve the monodispersity. ${ }^{20}$ Under pressure and with the aid of microwaves (MW), magnetite has also been synthesised by reduction of $\mathrm{Fe}\left(\mathrm{NO}_{3}\right)_{3}$ with ammonia in ethylene glycol at the gas-liquid interface. ${ }^{21}$ Morphology of iron oxide MNPs was shown to change with the type of solvent when synthesised in an autoclave. ${ }^{22}$

The use of an autoclave can also reduce the contribution of reaction kinetics dictated by temperature as the source of variation in nanoparticle size and composition. A study of FePt NP 
shape and size was performed in different solvents at the same temperature in an autoclave; rods, cubes and wires of different composition were obtained. ${ }^{\mathbf{1 4}}$ By limiting the freedom of molecules within a confined vessel, it is possible to employ temperatures above the normal boiling points of reagents. Solvents with different boiling points $\left(110-350{ }^{\circ} \mathrm{C}\right)$ were already employed in high pressure autoclave synthesis of FePt spheres, hexagons, cubes, rod and wire shaped MNPs. ${ }^{14}$ Elsewhere, autoclave assisted synthesis of crystalline $\mathrm{TiO}_{2} \mathrm{NPs}$ was reported for the first time. ${ }^{23}$ The use of pressure may also improve the crystallinity of FePt NPs. Ordered face centred tetragonal (fct) phase FePt has been synthesised under pressure (up to 6 bar) with the aid of MW using Collmans reagent, however it is not clear whether the MW or the pressure were the cause. ${ }^{24}$ Collmans redox reaction proceeds via a mutual oxidation and reduction of the respective salts of $\mathrm{Fe}^{2-}$ and $\mathrm{Pt}^{2+}$ to give metallic FePt, the balanced chemical equation is presented in Scheme 1.

Oleic acid (OA) is suggested to play four important roles; to act as proton source for protonation of acetylacetonate to acetylacetone; to coordinate with sodium to give an organic salt; to coordinate with Fe metal in its intermediate form and to stabilize the end product FePt MNPs. Oleylamine (OLA) is suggested to play two roles, to coordinate with Pt metal in its intermediate form and to stabilize the end product via dative bonds to Pt atoms on the surface of FePt NPs.

OA is shown to bind to magnetite MNPs with different energies, dependent on the size of the NP, and demonstrates the need for greater understanding of how MNPs are stabilised during the synthetic step. ${ }^{25}$ The surface chemistry of MNPs synthesised in organic media is also an important consideration for transferring them into aqueous media and further use in biomedicine, information on the functionalisation of MNPS can be found elsewhere. ${ }^{26,27}$

Here we present ellipsoids, cubes, and monkey nut shaped FePt NPs synthesised by varying individual OA and OLA amounts via the robust redox reaction of platinum(II) acetylacetonate with Collmans reagent on a Schlenk line and in an autoclave. The effect of pressure on this reaction type is investigated and shown to demonstrate potential for changing chemical composition and for subsequent properties of MNPs. Interactions between MNPs

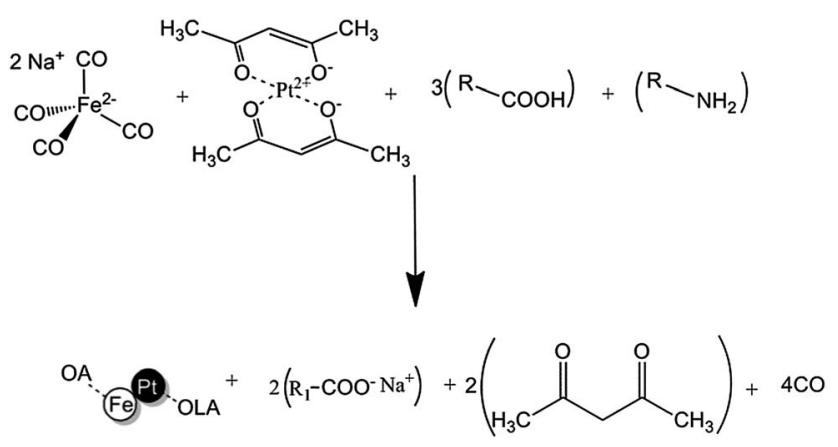

Scheme 1 Balanced equation for the reaction of Collmans reagent with platinum(II) acetylacetonate in the presence of oleic acid and oleylamine. and ligands are closely studied and offer useful information for the further functionalisation of MNPs.

\section{Results and discussion}

\section{Control of FePt nanoparticle morphology and stabilisation chemistry with surfactant amount on a Schlenk line}

Transmission electron microscopy (TEM) images shows cubic shaped NPs with low OLA amount (4 mmol) and monkey nut shaped MNPs (Fig. 1A and Fig. S1 $\dagger$ ) when OLA amount is increased to $12 \mathrm{mmol}$ on a Schlenk line (whilst maintaining total surfactant amount, $\mathrm{tSf}=16 \mathrm{mmol}$ and equiatomic metal precursors). The major diameters (determined as the circular outer circumferences of each particle ${ }^{28}$ ) increase (99\% level of confidence) with OLA amount from $7.4 \pm 0.8$ to $9.7 \pm 3.3 \mathrm{~nm}$, however minor diameters (determined as circular inner circumferences of each particle ${ }^{28}$ ) decrease (99\% level of confidence) from $6.2 \pm 0.6$ to $5.4 \pm 1.3 \mathrm{~nm}$ respectively. The aspect ratio (a measure of length, determined by the quotient of the major and minor diameters) increases to 2.2 and indicates the length of nanoparticles increases to approximately double the width (Fig. 1A). Cubic shapes are commonly observed in the syntheses of pure Pt NPs and large cubic FePt NPs with low Fe content are seen in the thermal decomposition synthesis of FePt MNPs. ${ }^{15,29}$

Diffraction patterns match closest to the pure platinum face centred cubic (fcc) phase (Fig. 1B and S2 $\dagger$ ). Crystallite sizes calculated using the Scherrer equation, indicate diameter increases from 6.6 to $8.7 \mathrm{~nm}$ with increasing OLA amount (Table S3 $\dagger$ ). The blocking temperature $\left(T_{\mathrm{B}}\right)$ increases $(21-67 \mathrm{~K}$ ) proportionally with the diameter of crystalline regions and aspect ratio. Diameters of crystalline regions determined using the Scherrer equation match closest to the TEM determined major diameters and suggest nanoparticles are composed of more crystalline than amorphous FePt alloy. Inductively coupled plasma (ICP) elemental analyses show increasing Fe content (10-14\%) and corresponding saturation magnetisation (0.02-1 emu.g $\left.{ }^{-1}\right)$ with increasing OLA amount (Table S4 $\dagger$ ). The actual saturation magnetisation will be higher than this due to the mass contribution of stabilising ligands but it will still be very low and incomparable to that of, for example magnetite

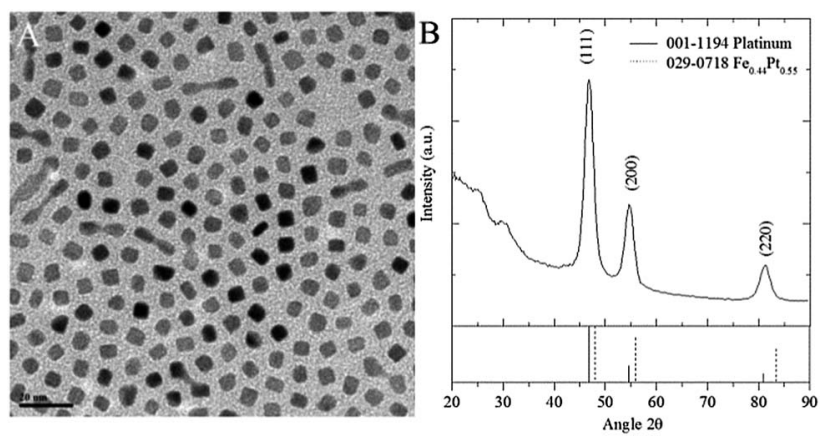

Fig. 1 A, TEM image and B, XRD diffraction pattern of cubes and monkey nut shaped NPs synthesised with OA : OLA ratio of $4: 12$ mmol. 
(91 emu.g ${ }^{-1}$ ). However the small increase shows that surfactant amount is important for control of NP Fe content and subsequent magnetic properties. Thermal decomposition and polyol reduction methods previously showed FePt MNPs with Pt rich cores and Fe rich shells. ${ }^{\mathbf{3 0 , 3 1}}$ Here, we see highly crystalline particles with similar size and low overall Fe content which indicates Pt rich particles similar to previous reports.

Under Schlenk conditions monkey nut shaped MNPs do not exhibit uniform width and appear to be the result of fused NPs, similar shaped rods have been seen before and are described as the result of an oriented attachment mechanism. ${ }^{32}$ Collmans reagent $\left(\mathrm{Na}_{2} \mathrm{Fe}(\mathrm{CO})_{4}\right)$ is a salt and insoluble in dioctyl ether whilst $\mathrm{Pt}(\mathrm{acac})_{2}$ is readily soluble, so it is suggested that oxidation of $\mathrm{Na}_{2} \mathrm{Fe}(\mathrm{CO})_{4}$ by $\mathrm{Pt}(\mathrm{acac})_{2}$ to give $\mathrm{Fe}^{0}$ could be inhibited by its insolubility in the reaction media. The slow release of $\mathrm{Fe}^{0}$ could lead to a similar mechanism and products to those seen in the polyol system. ${ }^{33}$ Monkey nut shaped MNPs are not seen when the reaction is performed in an autoclave here. However with a $\mathrm{Fe}: \mathrm{Pt}$ metal precursor ratio of $2: 1$, polydisperse and irregular shaped MNPs can be seen but are attributed to the absence of stirring (Fig. S3†). Another study further highlights the importance of the addition sequence and surfactant amount on FePt MNP size and shape. ${ }^{34}$ FePt rods have been seen in the literature when the injection of OLA is delayed $5 \mathrm{~min}$ after the injection of OA in the thermal decomposition of $\mathrm{Fe}(\mathrm{CO})_{5}$ with $\mathrm{Pt}(\mathrm{acac})_{2} \cdot{ }^{16}$ Increasing the amount of OLA has been shown to give monodisperse FePt NPs but they are irregularly shaped and exhibit decreasing diameter. This occurs due to a complex that forms between $\mathrm{Fe}(\mathrm{CO})_{5}$ and OLA; the presence of monkey nut shapes in our samples indicates that this process does not occur with Collmans reagent. ${ }^{32}$

FePt MNPs can be stabilised with the mono or bidentate forms of OA, and the mono dentate form of OLA, however it is unclear which synthetic parameters result in these different configurations. ${ }^{5,35}$ Shifts of specific peaks in Fourier transform infra-red (FTIR) spectra provide information about FePt NPstabilizer interactions. Upon synthesis with equiatomic metal precursor amounts, we observe a decrease in wavenumber of the symmetrical $\nu_{\mathrm{S}}\left(\mathrm{COO}^{-}\right)$from 1446 to $1394 \mathrm{~cm}^{-1}$ with increasing amount of OA (Fig. 2).

In its bi-dentate form, the carboxylic acid group of OA has a single electron shared across the two oxygen atoms, which it donates to Fe atoms on the surface of FePt NPs. We suggest the closer the carbonyl group is to the surface, the easier it is to donate an electron and coordinate with the metal surface. The result of this electron donation is that the $\mathrm{C}-\mathrm{O}$ bond becomes longer as the acid group now has less electron density to share between the carbon and oxygen atoms. This effect is analogous with hydrogen bonding between acids, except here, Fe metal behaves as recipient of electrons. ${ }^{36}$ With increasing amount of OA, FTIR shows the wavenumber of the $v_{\mathrm{s}}\left(\mathrm{COO}^{-}\right)$stretch decreases which gives information about the distance of the OA stabilizers from the surface of the MNP and the binding mode; Scheme 2 summarizes our observations.

We see strong $\nu_{\mathrm{S}}\left(\mathrm{COO}^{-}\right)$stretches $\left(1399 \mathrm{~cm}^{-1}\right)$ and asymmetrical stretches $\nu_{\mathrm{A}}\left(\mathrm{COO}^{-}\right)$stretches $\left(1591 \mathrm{~cm}^{-1}\right)$ when OA is at a maximum $(\mathrm{OA}: \mathrm{OLA}=12: 4)$, these peaks are weak when

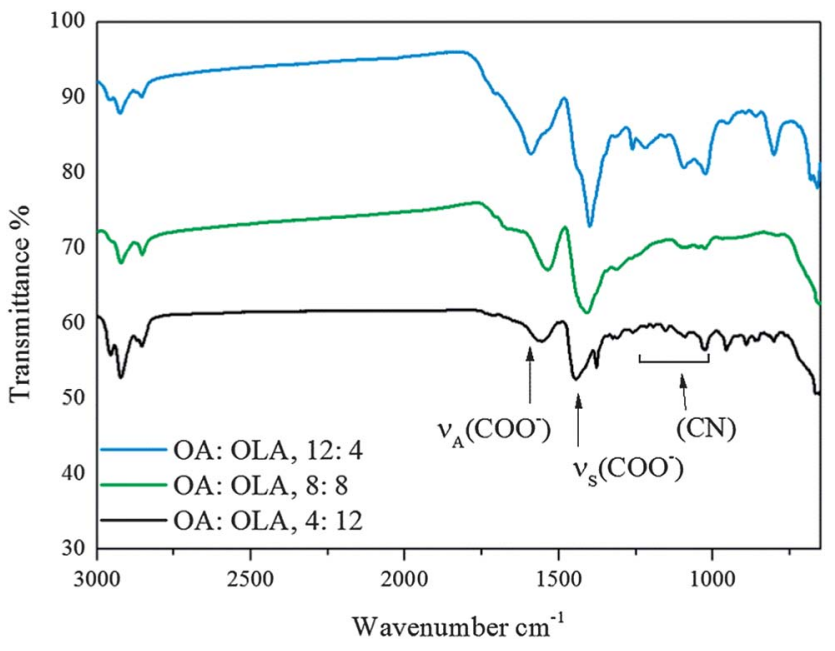

Fig. 2 FTIR spectra of samples 1-3, synthesised under Schlenk conditions with equiatomic Fe and Pt metal precursor amounts.

$\mathrm{OA}$ is a minimum (OA : OLA $=4: 12$, Fig. 2$) \cdot{ }^{36} \alpha \beta$-unsaturation of carbonyl groups causes a lowering of the $\mathrm{C}=\mathrm{O}$ frequency by up to $60 \mathrm{~cm}^{-1}$ and hydrogen bonding can cause a lowering of up to $40 \mathrm{~cm}^{-1}$, totalling to a net effect of $100 \mathrm{~cm}^{-1}$ (ref. 36). The decreasing wavenumber of the $v_{\mathrm{S}}\left(\mathrm{COO}^{-}\right)$symmetrical mode with increasing amount of $\mathrm{OA}$ indicates single $\mathrm{C}-\mathrm{O}$ bonds are longer when OA is readily available and can easily coordinate with the FePt surface and that $\mathrm{OA}$ is the main stabilizing surfactant.

In the case of mono-dentate binding, both a single $\mathrm{C}-\mathrm{O}$ bond and a double $\mathrm{C}=\mathrm{O}$ bond will be present. The double $\mathrm{C}=\mathrm{O}$ bond will be stronger and exhibit higher wavenumber when there is a higher probability of asymmetric stretching. The increase in wavenumber of the $v_{\mathrm{A}}\left(\mathrm{COO}^{-}\right)$asymmetric mode with increasing OA confirms a stronger double $\mathrm{C}=\mathrm{O}$ bond which supports the case for mono-dentate binding of OA.

Peaks in the range $1095-1263 \mathrm{~cm}^{-1}$ result from the $\mathrm{C}-\mathrm{N}$ stretch, with low OA amount these peaks vary across this range, while with high OA amount we see a prominent peak at $1263 \mathrm{~cm}^{-1}$, which corresponds to the $\mathrm{C}-\mathrm{N}$ bond. Strong $\mathrm{NH}_{2}-\mathrm{Pt}$ interactions will exhibit longer $\mathrm{C}-\mathrm{N}$ stretches with lower

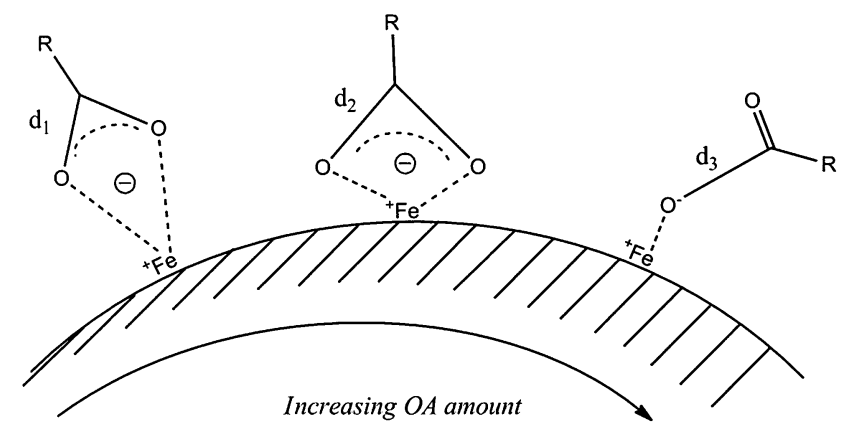

Scheme 2 Different binding modes of OA with the FePt nanoparticle surface, $d$ represents the length of the bond; $d_{1}<d_{2}<d_{3}, \nu_{\mathrm{S}}\left(\mathrm{COO}^{-}\right)$ wavenumber decreases from $1446-1396 \mathrm{~cm}^{-1}$ when the amount of $\mathrm{OA}$ is increased from 4 to $12 \mathrm{mmol}$. 
wavenumber due to the dative bond formed between the nitrogen lone pair of electrons and $\mathrm{Pt}$, while weakly bound $\mathrm{NH}_{2}$ will exhibit shorter $\mathrm{C}-\mathrm{N}$ stretches with higher wavenumber due to less donation of electron density to the dative bond.

When Fe: Pt precursor ratio is increased to $2: 1$, the $\nu_{\mathrm{S}}\left(\mathrm{COO}^{-}\right)$stretch is observed at $1427 \mathrm{~cm}^{-1}$ and suggests bidentate OA stabilizes these NPs when a low amount of OA is employed (OA : OLA $=4: 12 \mathrm{mmol}$ ). As the amount of OA is decreased from 12 to $8 \mathrm{mmol}$, the peak for mono-dentate OA $\left(\sim 1672 \mathrm{~cm}^{-1}\right)$ disappears and indicates that OA is no longer coordinated in a mono-dentate configuration. Instead, a stretch typical of bi-dentate OA is present at $1427 \mathrm{~cm}^{-1}$ and a peak at $1563 \mathrm{~cm}^{-1}$ indicates the presence of either $\nu_{\mathrm{A}}\left(\mathrm{COO}^{-}\right)$or the scissoring mode of $\mathrm{NH}_{2}$, however, further presence of peaks at 1069 , and $842 \mathrm{~cm}^{-1}$ support the case for the $\mathrm{NH}_{2}$ group. ${ }^{35}$ With further decrease in OA amount from 8 to $4 \mathrm{mmol}$ the $\nu_{\mathrm{S}}\left(\mathrm{COO}^{-}\right)$stretch at $1427 \mathrm{~cm}^{-1}$ and the $(\mathrm{N}-\mathrm{H})$ bend at $880 \mathrm{~cm}^{-1}$ become the clear features, however there are minor peaks at 1672, 1563, 1336 and $1069 \mathrm{~cm}^{-1}$, the presence of all these peaks suggests a mixture of mono and bi-dentate OA and mono-dentate $\mathrm{NH}_{2}$.

The effect of surfactant amount is fairly similar under Schlenk line reactions to those in an autoclave. With equiatomic FePt, all samples exhibit peaks which correspond to the stretches and bends of mono and bi-dentate OA, and OLA. Doubling the amount of Fe metal precursor (Fe : Pt $=2: 1$ ) leads to only OA being responsible for stabilizing the particles. Fe is commonly coordinated by OA, so it is suggested that there is more Fe at the surface of NPs when the Fe metal precursor amount is doubled. ${ }^{6}$

Changes in binding energy of OA with different sized magnetite MNPs were previously reported and attributed to a balancing of the Van der Waals forces and repulsion forces; ${ }^{25}$ here, it is suggested that the binding energies might be attributed to the binding modes instead. The surface chemistry can be controlled by two approaches, firstly by changing the ratio of OA to OLA and secondly by changing the Fe : Pt metal precursor amount ratio. Stretches and bends associated with the $\mathrm{NH}_{2}$ group are present with high amount of OLA and are seen in conjunction with the bi-dentate form of OA. By doubling the amount of Fe precursor, a bi-dentate configuration of OA and mono-dentate configuration of OLA are favoured; there is no evidence for amine stabilization when $8 \mathrm{mmol}$ or less of OLA is employed.

\section{Impact of Fe precursor amount on morphology, Fe content and crystallography of FePt nanoparticles}

Iron precursor $\left(\mathrm{Na}_{2} \mathrm{Fe}(\mathrm{CO})_{4}\right)$ amount was doubled in an attempt to increase the Fe content of our FePt NPs. Under Schlenk conditions, minor diameters statistically increase, while the standard deviation decreases (Table S3†, samples 9, 11, 13). Cubic and ellipsoid shapes are retained when syntheses were performed on a Schlenk line, however sizes determined with the Scherrer equation predominantly match closer to the minor diameters (determined by TEM) which suggests that the crystalline region of these nanoparticles is smaller than the overall size and could indicate the presence of an amorphous shell.

Equivalent diameter of FePt NPs remains approximately the same when the Fe metal precursor amount is doubled under autoclave conditions (Table S3†, samples, 19, 21, and 23) however, cubes have rounder edges and are modelled more efficiently with Pebbles software, ${ }^{28}$ using an ellipsoid shape. Unlike those synthesised under Schlenk conditions, they retain crystalline regions with diameters that match closest to the major diameters of FePt NPs.

With increasing OLA amount (4-12 mmol) Fe content of NPs increases from 10 to $14 \%$ under Schlenk conditions and from 4 to $24 \%$ under autoclave conditions. Doubling the Fe metal precursor amount increases these ranges from 12 to $20 \%$ and from 10 to $36 \%$ under Schlenk and autoclave conditions respectively.

Saturation magnetisation values correspondingly increase from approximately $0.0-1.0 \mathrm{emu}^{\mathrm{g}} \mathrm{g}^{-1}$ to $0.1-3.1 \mathrm{emu}^{\mathrm{g}} \mathrm{g}^{-1}$ under Schlenk conditions and from $0.5-4.9$ emu.g $^{-1}$ to $1.0-$ 25.1 emu.g $^{-1}$ under autoclave conditions (Table S4 $\dagger$ ). The shapes of hysteresis loops are linear and paramagnetic in shape with high amount of OA; as the amount of OA is decreased, loops become more $\mathrm{S}$ shaped indicating evidence for superparaor ferro-magnetism (Fig. S6†).

\section{Changes of Fe content and saturation magnetisation with the use of an autoclave}

The properties of FePt MNPs resulting from reaction of Collmans reagent with $\mathrm{Pt}(\mathrm{acac})_{2}$ on a Schlenk line (samples 7-16, Table $\mathrm{S} 1 \dagger$ ) were compared to those synthesised in a sealed autoclave (samples 17-26, Table S1 $\dagger$ ). Previously, a low ratio of OA to OLA was shown to result in higher Fe content. With a ratio of Collmans reagent to platinum acetylacetonate of $2: 1$, the effect of surfactant amounts on morphology, crystallography, Fe content and magnetic properties on a Schlenk line and in an autoclave are further investigated here.

The majority of FePt NPs exhibit a cubic shape (Table S3 and Fig. $\mathrm{S} 4 \dagger$ ) however monkey nut shaped MNPs with aspect ratio up to 2.2 are also seen when a high amount of OLA is employed (sample 1, Fig. S1C ${ }^{\dagger}$ ) on a Schlenk line. Here, there is no general trend of FePt NP size with increasing amount of OLA. However, the diameters of crystalline regions (determined by the Scherrer equation) appear to decrease overall from 6.5 to 3.6 and match closest to the TEM determined minor diameters (Table S3 $\dagger$ ). XRD patterns all exhibit the fcc phase and match closest to a platinum reference (ref. 001-1194) however there is a shift from 46.8 to $47.0^{\circ} 2 \theta$ with increasing amount of OLA, though not significant, an explanation is offered. Braggs law $(\lambda=2 d \sin \theta)$ dictates that with smaller $d$ spacings, higher angles of diffraction are expected. The distance between (111) lattice planes is $2.20 \AA$ for $\mathrm{Fe}_{0.44} \mathrm{Pt}_{0.55}$ and $2.25 \AA$ for pure Pt. With a higher percentage of Fe atoms (which are smaller than Pt atoms), more Fe-Fe interactions will be present and decrease the average lattice parameter of the crystallite and increase the angle $2 \theta$ at which diffraction peaks are found. Saturation magnetization 
and blocking temperature increase from 0.1 to 14.6 emu.g $^{-1}$ and from 16 to $88 \mathrm{~K}$ respectively when the amount of OLA is increased from 0 to $16 \mathrm{mmol}$ (Table S4 and Fig. S8†). ICP supports these data with an increase of Fe content from 4 to $21 \%$ as OLA is increased from 4 to $12 \mathrm{mmol}$.

TEM shows cubic MNPs with rounder edges when the syntheses were performed in an autoclave compared to those synthesised at normal pressure; no monkey nut shapes or cubes with aspect ratio greater than 1.3 were observed (Table S3 and Fig. S5 $\dagger$ ). Similar to MNPs synthesised on the Schlenk line, there is no trend in size with increasing OLA amount. However, unlike those synthesised on a Schlenk line, the diameters of crystalline regions (determined by the Scherrer equation) within FePt NPs match closer to the major diameters determined by TEM and decrease overall from 6.5 to $4.4 \mathrm{~nm}$. This suggests that NPs synthesised under pressure are more crystalline than those synthesised under normal pressure. The increase of crystallinity is in agreement with the observation made when crystalline titania NPs were synthesised for the first time in an autoclave. ${ }^{23}$ XRD shows fcc phase FePt and when synthesised with a high amount of OLA, there is evidence for iron oxide (Fig. S2 $\dagger$ ). Similar to syntheses on a Schlenk line, ICP shows an increase of Fe content from 10 to $35 \%$ and magnetic saturation and blocking temperature increase as OLA amount is increased from 4 to $12 \mathrm{mmol}$ in an autoclave (Table S4 and Fig. S8†).

MNPs synthesised in an autoclave have greater standard deviation from the equivalent mean diameter than those synthesised under normal pressure and no monkey nut shapes are observed. In the synthesis of FePt NPs it is desirable to achieve nucleation of all NPs in a short time frame in order to prevent the onset of ripening mechanisms and broadening of polydispersity. ${ }^{37}$ On the Schlenk line, stirring was performed using a magnetic stirrer, however due to the nature of our apparatus it was impossible to set up a stirring mechanism for the autoclave. The authors suspect that the lack of stirring led to the range of shapes observed and broadening of polydispersity. Aggregation

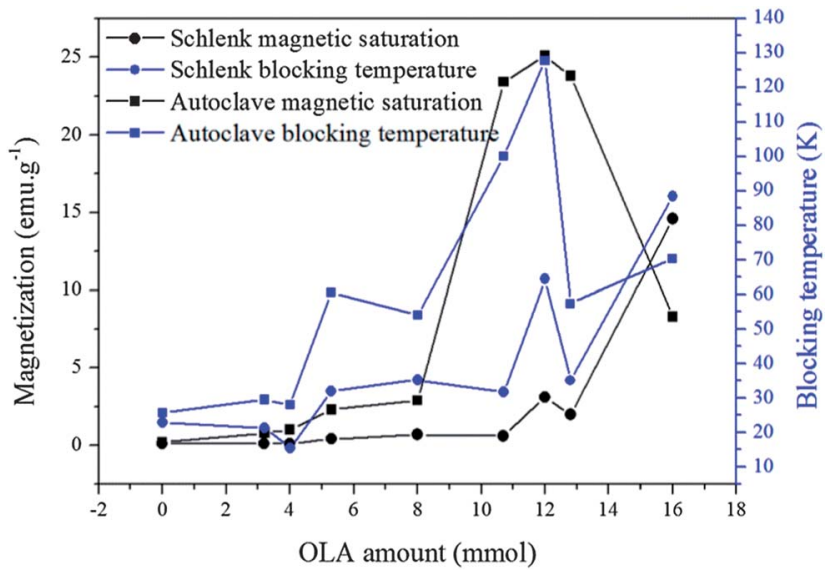

Fig. 3 Saturation magnetisation and blocking temperature of samples synthesised on a Schlenk line (from left to right, samples 7-15) and in an autoclave (from left to right, sample 17-25). The data were extracted from the hysteresis loops and ZFC-FC curves in Fig. S8. $\dagger$ is observed when no OA is employed under both Schlenk line and autoclave conditions.

$M_{\mathrm{S}}$ and $T_{\mathrm{B}}$ of FePt MNPs both appear to be higher when the syntheses are performed under autoclave conditions than on a Schlenk line, however further tests are required to confirm this. The exception to this rule is when OLA is at a maximum, $M_{\mathrm{S}}$ and $T_{\mathrm{B}}$ of MNPs synthesised in an autoclave are lower than those synthesised on a Schlenk line when OLA $=16 \mathrm{mmol}$ (Fig. 3). However, XRD of FePt NPs synthesised in an autoclave showed some magnetite peaks, though their intensities are significantly lower than those observed for the FePt fcc phase. The presence of magnetite could be attributed to lack of stirring leading to inhomogeneity of the reactants and incomplete reaction of the Fe precursor. Upon completion, exposure to air could result in the remaining unreacted Collmans reagent oxidising to give magnetite.

\section{Conclusion}

FePt cubes and monkey nut shaped NPs were synthesised in a controlled manner on a Schlenk line and in an autoclave using Collmans reagent for the first time. In general FePt NPs exhibit higher Fe content and $M_{\mathrm{S}}$ (up to $35 \%$ and 25.1 emu.g ${ }^{-1}$ with $12.8 \mathrm{mmol}$ OLA) when synthesised in an autoclave than on a Schlenk line (up to $21 \%$ and 14.6 emu.g $^{-1}$ respectively with $12.8 \mathrm{mmol}$ OLA).

Increasing OLA amount (from 4 to $12 \mathrm{mmol}$ ) on a Schlenk line with constant total surfactant amount $(16 \mathrm{mmol})$ yielded monkey nut shaped NPs with an aspect ratio up to 2.2 and increased Fe content (10-14\%) and saturation magnetisation; Fe content and $M_{\mathrm{S}}$ both increase under the same conditions in an autoclave.

Doubling the amount of Collmans reagent leads to FePt NPs with higher Fe content. Nano cubes synthesised in an autoclave have larger crystalline regions than those synthesised on a Schlenk line. Binding modes of OA to the surface of NPs can be changed through the use of different amounts of OA and OLA. Mono-dentate configuration of OA and OLA is preferred with large amounts of OLA whilst bi-dentate configuration of OA occurs with large amounts of OA.

We have demonstrated the effects of pressure and surfactant amounts on the fabrication of FePt MNPs when synthesised with Collmans reagent and platinum acetylacetonate. In previous syntheses of FePt MNPs, maintaining Fe content while increasing MNP size was a challenge; we have shown the introduction of pressure can increase the Fe content and corresponding magnetic properties. Increasing OLA under Schlenk conditions has been shown to encourage the oriented-attachment mechanism to give elongated FePt, whilst lack of stirring under autoclave conditions led to dominance of the classic Ostwald ripening mechanism.

Further investigation of the effect of stirring in an autoclave is being pursued, however it is expected that the effect of pressure will continue to be researched as a parameter for controlling the elemental composition of NPs. These results may help to guide research in the synthesis of FePt NPs with improved properties. Characterisation of FePt NPs with infra- 
red spectroscopy is rarely reported, it is envisaged that the important shifts in spectra observed here, will also aid in choosing appropriate subsequent functionalisation routes after synthesis. Ultimately, through the use of pressure and careful tuning of stabiliser amount, the application of next generation FePt $\mathrm{NPs}^{6}$ in biomedicine and other applications has taken a step forward.

\section{Acknowledgements}

Nguyễn T. K. Thanh would like to thank the Royal Society for her University Research Fellowship; Luke Green would like to thank UCL for his PhD studentship.

\section{Notes and references}

1 Q. A. Pankhurst, J. Connolly, S. K. Jones and J. Dobson, J. Phys. D: Appl. Phys., 2003, 36, R167-R181.

2 K. M. Krishnan, IEEE Trans. Magn., 2010, 46, 2523-2558.

3 P. A. Xu, G. M. Zeng, D. L. Huang, C. L. Feng, S. Hu, M. H. Zhao, C. Lai, Z. Wei, C. Huang, G. X. Xie and Z. F. Liu, Sci. Total Environ., 2012, 424, 1-10.

4 Y. H. Zhu, L. P. Stubbs, F. Ho, R. Z. Liu, C. P. Ship, J. A. Maguire and N. S. Hosmane, ChemCatChem, 2010, 2, 365-374.

5 H. W. Zhang, Y. Liu and S. H. Sun, Front. Phys. China, 2010, 5, 347-356.

6 N. T. K. Thanh, Magnetic nanoparticles: from fabrication to clinical applications: theory to therapy, chemistry to clinic, bench to bedside, CRC, Taylor \& Francis [distributor], London, Boca Raton, Fla., 2012.

7 R. M. Taylor, D. L. Huber, T. C. Monson, A. M. S. Ali, M. Bisoffi and L. O. Sillerud, J. Nanopart. Res., 2011, 13, 4717-4729.

8 S. H. Sun, C. B. Murray, D. Weller, L. Folks and A. Moser, Science, 2000, 287, 1989-1992.

9 Q. A. Pankhurst, N. K. T. Thanh, S. K. Jones and J. Dobson, J. Phys. D: Appl. Phys., 2009, 42, 224001.

10 S. Maenosono and S. Saita, IEEE Trans. Magn., 2006, 42, 1638-1642.

11 M. S. Seehra, V. Singh, P. Dutta, S. Neeleshwar, Y. Y. Chen, C. L. Chen, S. W. Chou and C. C. Chen, J. Phys. D: Appl. Phys., 2010, 43, 145002.

12 S. Maenosono, T. Suzuki and S. Saita, J. Magn. Magn. Mater., 2008, 320, L79-L83.

13 C. Liu, X. W. Wu, T. Klemmer, N. Shukla, X. M. Yang, D. Weller, A. G. Roy, M. Tanase and D. Laughlin, Abstr. Pap. Am. Chem. Soc., 2004, 227, 936, INOR.

14 N. Shukla, M. M. Nigra, T. Nuhfer, M. A. Bartel and A. J. Gellman, Nanotechnology, 2009, 20, 065602.
15 D. Ung, L. D. Tung, G. Caruntu, D. Delaportas, I. Alexandrou, I. A. Prior and N. T. K. Thanh, CrystEngComm, 2009, 11, 1309-1316.

16 V. Nandwana, K. E. Elkins, N. Poudyal, G. S. Chaubey, K. Yano and J. P. Liu, J. Phys. Chem. C, 2007, 111, 41854189.

17 B. R. Cuenya, Acc. Chem. Res., 2013, 46, 1682-1691.

18 R. E. Serda, B. Godin, E. Blanco, C. Chiappini and M. Ferrari, Biochim. Biophys. Acta, Gen. Subj., 2011, 1810, 317-329.

19 Y. C. Zhu, T. Mei, Y. Wang and Y. T. Qian, J. Mater. Chem., 2011, 21, 11457-11463.

20 A. L. Andrade, M. A. Valente, J. M. F. Ferreira and J. D. Fabris, J. Magn. Magn. Mater., 2012, 324, 1753-1757.

21 Z. M. Cui, L. Y. Hang, W. G. Song and Y. G. Guo, Chem. Mater., 2009, 21, 1162-1166.

22 F. J. Douglas, D. A. MacLaren and M. Murrie, $R S C A d v ., 2012$, 2, 8027-8035.

23 P. Hald, J. Becker, M. Bremholm, J. S. Pedersen, J. Chevallier, S. B. Iversen and B. B. Iversen, J. Solid State Chem., 2006, 179, 2674-2680.

24 H. L. Nguyen, L. E. M. Howard, S. R. Giblin, B. K. Tanner, I. Terry, A. K. Hughes, I. M. Ross, A. Serres, H. Burckstummer and J. S. O. Evans, J. Mater. Chem., 2005, 15, 5136-5143.

25 L. Zhang, R. He and H. C. Gu, Appl. Surf. Sci., 2006, 253, 2611-2617.

26 N. T. K. Thanh and L. A. W. Green, Nano Today, 2010, 5, 213230.

27 H. G. Bagaria, E. T. Ada, M. Shamsuzzoha, D. E. Nikles and D. T. Johnson, Langmuir, 2006, 22, 7732-7737.

28 S. Mondini, A. M. Ferretti, A. Puglisi and A. Ponti, Nanoscale, 2012, 4, 5356-5372.

29 J. Y. Chen, B. Lim, E. P. Lee and Y. N. Xia, Nano Today, 2009, 4, 81-95.

30 M. Delalande, P. R. Marcoux, P. Reiss and Y. Samson, J. Mater. Chem., 2007, 17, 1579-1588.

31 S. H. Sun, Adv. Mater., 2006, 18, 393-403.

32 B. Bian, W. Xia, J. Du, J. Zhang, J. P. Liu, Z. Guo and A. Yan, Nanoscale, 2013, 5, 2454-2459.

33 N. Poudyal, G. S. Chaubey, C. B. Rong and J. P. Liu, J. Appl. Phys., 2009, 105, 07A749.

34 D. A. Ferrer, S. Guchhait, H. Liu, F. Ferdousi, C. Corbet, H. Xu, M. Doczy, G. Bourianoff, L. Mathew, R. Rao, S. Saha, M. Ramon, S. Ganguly, J. T. Markert and S. K. Banerjee, J. Appl. Phys., 2011, 110, 014316.

35 N. Shukla, C. Liu, P. M. Jones and D. Weller, J. Magn. Magn. Mater., 2003, 266, 178-184.

36 D. H. Williams and I. Fleming, Spectroscopic methods in organic chemistry, McGraw-Hill, London, 1995.

37 P. J. Skrdla, J. Phys. Chem. C, 2012, 116, 214-225. 